\title{
Design and Implementation of Medical Negative Pressure Machine Monitoring System Based on the Internet of things
}

\author{
Sun Ling, Ji Hongwei, Bu ran, Tang Zhiyong, Li Xinrui \\ School of electronic and information engineering, Nantong University, Nantong 226019, China \\ Corresponding Author: Sun.I@ntu.edu.cn
}

Key words: Internet of things, touch control, medical negative pressure machine, sensor

\begin{abstract}
An automatic monitoring system which is based on the Internet of things is designed to resolve the singleness of function and of agility of the traditional system. The system is constituted by several modules of pressure, water level, temperature, serial communication and touch screen control. The device can collect the real time data and transmit those to the Micro control chip and then finish the functions of implementation touch parameters setting, flip, pump swap, alarm and others by the touching screen. The project is promising and will play a great role later on.
\end{abstract}

\section{Introduction}

The negative pressure is mainly used for the suction of sputum, blood, pus and other pollutants in the patient's body ${ }^{[1]}$. However, most of the medical negative pressure machines' intelligence are not convenient and the machine can cause risks. So it is necessary to design an automatic monitoring system which can behave better. This system can save energy greatly and improve the life of the vacuum pump. The technology is involved in the local network or the Internet and other communication technologies to connect people and things together in a new way, which is made use of to realize the real-time monitoring.

\section{Design of The overall framework}

The overall design of the automatic monitoring system is shown in figure 1. One block is the negative pressure machine and the other is the water tank. The negative pressure machine takes control of the pressure in the vacuum tank and the water tank provides circulating water that the negative pressure machine needs. The system uses double pump rotation mode. The SCM controls negative pressure machine to exchange work and the pressure sensor transmits the real-time value back to the SCM. Then, the SCM controls pressure according to the value of pressure and the upper and the lower limits. The data of temperature sensor and water level sensor will be transmitted back to the SCM. When the temperature exceeds the preset value, the SCM starts cooling process. The related data packets are transmitted to the user's PC through w5500 and the user can monitor the condition of the system ${ }^{[2]}$.

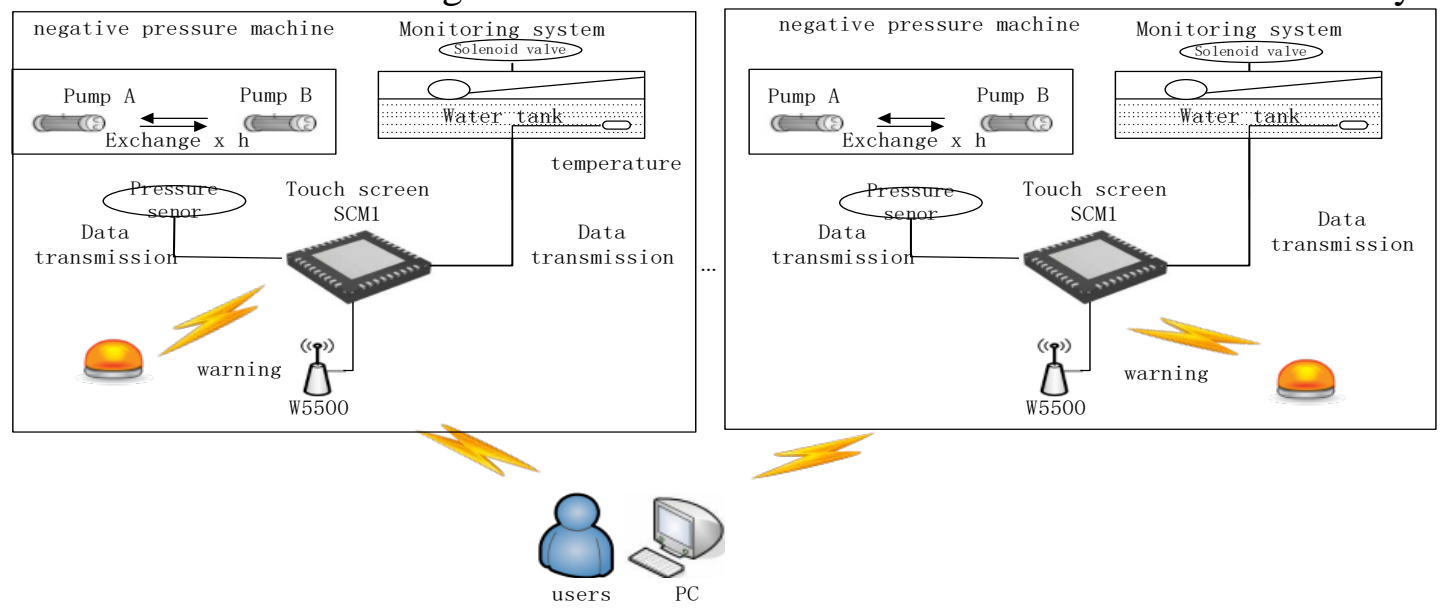

Figure 1 Overall architecture diagram of the negative pressure monitoring system 


\section{The Design of Hardware}

2.1 Hardware Design Framework.The automatic monitoring system of the negative pressure machine mainly consists of the SCM, clock, power, pressure sensor, water level sensor, water temperature sensor, relay, W5500 serial port communication and touch screen.

2.2 The Design of PCB.The design of PCB is achieved by the software called Altium Designer. Firstly, draw the schematic diagram of the data collective system in Altium Designer software. Then export the components to the PCB layout. Finally, wire the components in the layout of the PCB. The PCB circuit board which has welded is shown in figure 3.

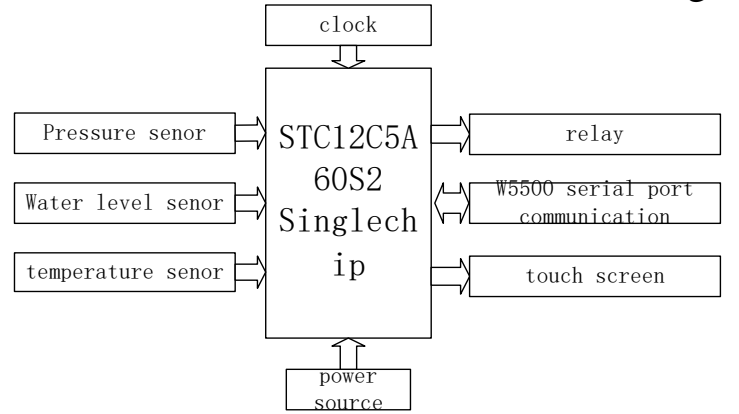

Figure 2 the design of hardware

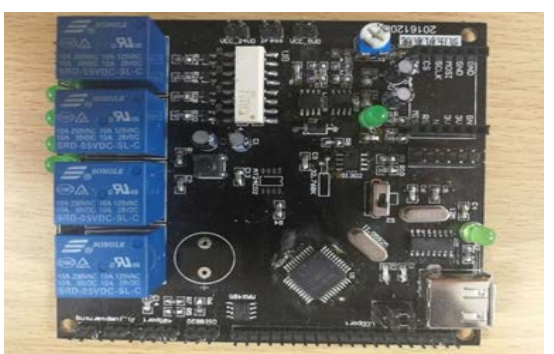

Figure 3 the design of PCB

2.3 I/V Transform Circuit.The output signal of the pressure sensor is current ,but the SCM in the system can only deal with the problem of voltage signal. So a current voltage (I-V) conversion circuit is designed. According to the requirement of the system, this paper designs an active $\mathrm{I} / \mathrm{V}$ conversion circuit as shown in figure 4. In Figure 4, the circuit is firstly used to complete the conversion of the current voltage signal by the resistor R1; the dashed line shows a low pass filter which is composed of a resistor $\mathrm{R} 2$ and a capacitor $\mathrm{C} 1$. If the cut-off frequency of the filter is $50 \mathrm{~Hz}$, according to the cutoff frequency formula $\mathrm{H}=1$ / ( $\mathrm{RC}$ ) of the low-pass filter, the value of the resistor $\mathrm{R} 2$ and the capacitance C1 can be determined. Capacitor C2 and C3 is a decoupling capacitor and the capacitor C2 also provides local DC power supply for operational amplifier U2A. Resistance R5 is to prevent the op-amp output short circuit. The voltage amplifying circuit composed of operational amplifier LM358 is used to amplify the voltage signal on both ends of R1 so as to realize the conversion of current signal to voltage signal.

In Figure 4, assuming the output current of sensor is I and the voltage at the ends of the resistor R1 is

$$
V_{R 1}=I \times R_{1}
$$

The operational amplifier LM358 and the resistance R3, R4 are composed of a voltage amplification circuit. According to the connection of the circuit and the characteristics of "virtual short" and "virtual open" of the operational amplifier, The output voltage of voltage amplification circuit is

$$
\text { Vout }=I \times R_{1} \times\left(1+\frac{R_{4}}{R_{3}}\right)-V_{R E F} \times \frac{R_{4}}{R_{3}}
$$

This shows that resistance R1, R3 and R4 choose the appropriate resistance to complete a practical application of the current voltage conversion circuit.

Figure 5 gives the emulational image of I/V conversion circuit. From the picture, the 4 to $20 \mathrm{~mA}$ current signal and the 0 to $5 \mathrm{~V}$ voltage signal are linear. This means the $\mathrm{I} / \mathrm{V}$ conversion circuit is suitable for our system.
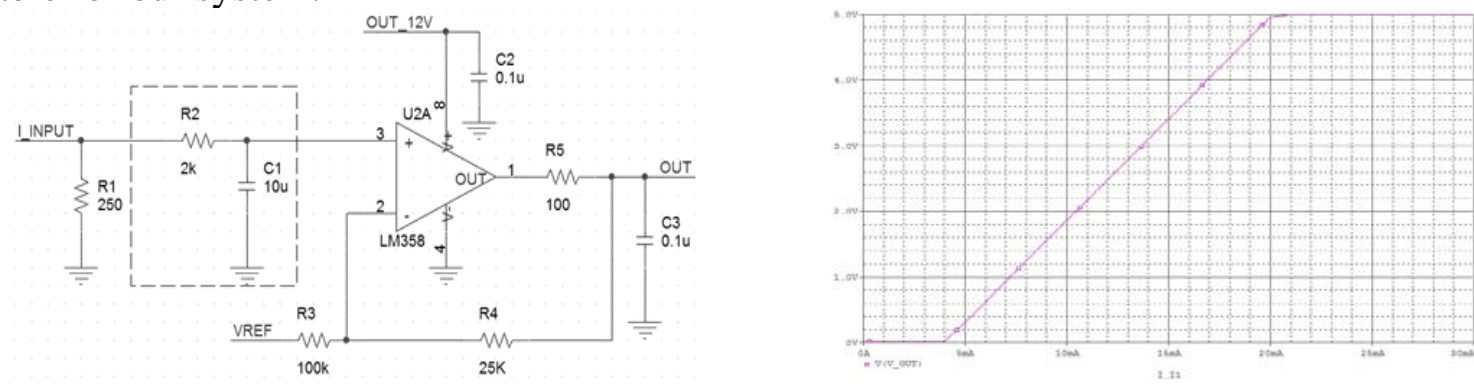

Figure 4 the principle diagram of limiting circuit Figure 5 emulational image of I/V conversion circuit 
2.4 W5500 Serial Communication.The W5500 chip is a Hardwired TCP/IP embedded Ethernet controller that provides convenient Internet connection to embedded systems. W5500 enables users to have the Internet connectivity in their applications just by using the single chip in which TCP/IP stack, 10/100 Ethernet MAC and PHY embedded.

The W5500 embeds the 32Kbyte internal memory buffer for the Ethernet packet processing. And, W5500's SPI supports $80 \mathrm{MHz}$ speed and new efficient SPI protocol for the high speed network communication. So, it's a more faster and easier chip to use than using any other Embedded Ethernet solution.

\section{Software Design}

3.1 Master Control Program. The master control program is mainly used for taking the control of the pump A and B , water level, pressure control, setting time, detection of water temperature and altitude. The once control process is shown in figure 6. Once a process is completed, loopingthis process[3].
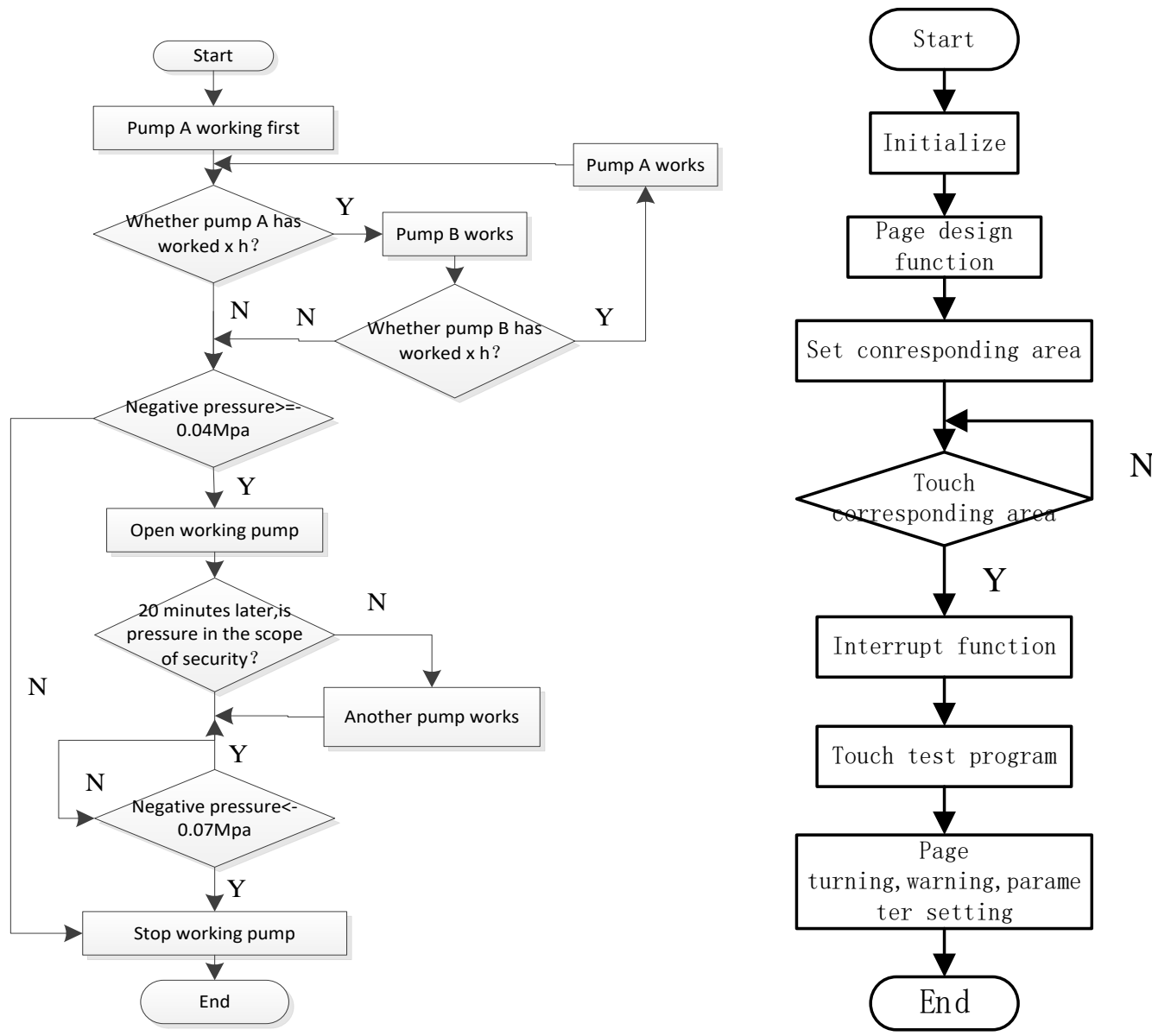

Figure 6 once control process

Figure 7 the design process of touch screen

3.2 Design and Realize of Touch Screen Drivers. There are 8 pages in this system (one main page and seven flowing pages) which are divided into 8 flowing programs for calling. Setting the coordination by locating the position of character string. The water temperature and pressure display functions were used to show the water temperature and pressure[4].

\section{Test Result and Functions Showing}

System testing:

First of all, the system sets the initial parameters of field test.

parameter 1 : the upper and the lower negative pressure limits are respectively $-0.040 \mathrm{Mpa}$, $-0.070 \mathrm{Mpa}$ 
parameter 2: pump swapping time: 2 minutes; parameter 3: timeout limit: 20 minutes;

parameter 4: water temperature limit: $25^{\circ} \mathrm{C}$, water exchange time: 10 days;

parameter 5: water level limit: 0.110M, median level limit: 0.050M, water level limit: 0.010M.

Then, starting the test of the pressure, water level and water temperature control system performance. The measurement process is followed:

Pressure control of pump A and B interchange process:

1)When the system's time is not up to 2 minutes, the pump A is working; the pump B is waiting. When the pressure is less than the set value, open the pump A to increase the pressure up to the upper limit and then the pump $\mathrm{A}$ is waiting.

2)When the system's time is up to 2 minutes, the pump B is working; the pump A is waiting. When the pressure is less than the set value, open the B pump to increase the pressure up to the upper limit and then the pump B is waiting.

3)When the system's time is up to 2 minutes, change the pump A as the working pump. So the pump A and B cycle use.

4)When the pressure is higher than the upper limit for twenty minutes, the two pumps work at the same time

The control process of water level and temperature:

1)When the water temperature exceeds $25^{\circ} \mathrm{C}$, open the drain valve of water first; until the water level reached the median $0.050 \mathrm{M}$, close the drain valve and open the inlet valve;close the drain valve until the water level reaches the high value of $0.110 \mathrm{M}$; If the water temperature is still more than $25^{\circ} \mathrm{C}$, repeat the operation.

2)When the water exchange time reached 10 days, began to change the water tank. Open the drain valve first until the water level reaches the low value of $0.010 \mathrm{M}$; close the drain valve and open the inlet valve; close the inlet valve until the water level reaches the high value of $0.110 \mathrm{M}$.

The system works well and can realize the function that is needed.

\section{Conclusion}

The overall system architecture is secure and stable., the pressure module,the water level and water temperature and the communication software collocation is reasonable, accurate and efficient while ensuring the normal operation. This saves the cost and improves the promotion of the value. With the development of sensor technology and the expansion of the use of the medical negative pressure, this will add the new vigor for the development of the industry and meet people's needs.

\section{Acknowledgements}

This work was supported by the Production and Research Technology Projects of Jiangsu Province (BY2016053-08), and the Innovation Training Program for college students(201610304088X).

\section{References}

[1]Red Zhu Qinglin, Jia, Ao Faliang. Design PC and single-chip computer real-time communication system and implementation of [J]. micro computer information, 2007, (02):143-145-200.

[2]Guo Jia, Zhou Miaomiao. Wireless temperature monitoring system based on DS18B20 for dry type transformer [J]. electrical measurement and instrumentation, 2014, (22):92-96.

[3]Guo Xiang, Wang Keying, Li Jing. Design and implementation of automatic test equipment for power transformer based on AVR MCU [J]. electric measurement and instrument, 2012, (08):71-74. [4]Shaw. Jingwen RA8806 controller touch screen design based on [J]. control and instruments in chemical industry, 2014, (10):1211-1213.

[5] Mumtaz S., Alsohaily A., Pang Z.Massive Internet of Things for Industrial Applications[J]. IEEE Industrial Electronics Magazine,2017,11(1):28-33. 